\title{
LA FINANCIARIZACIÓN DE LA AGRICULTURA: LA PARTICIPACIÓN DEL PLAN DE PENSIONES DE CANADA EN GLENCORE
}

\author{
Flavia Echánove Huacuja ${ }^{1}$ \\ Universidad Nacional Autónoma de México (UNAM) ${ }^{2}$
}

\begin{abstract}
RESUMEN
La financiarización del sistema global alimentario, intensificada a raíz de la crisis del 2007-08, ha dado lugar a la diversificación y el fortalecimiento de los llamados agentes financieros. Entre éstos se encuentran los inversores institucionales, que agrupan a entidades como los fondos de pensiones (privados y públicos). Entre las estrategias de inversión de éstos, han adquirido relevancia las realizadas en el sector agro-alimentario, tanto en las distintas fases de las cadenas de valor (acopio, producción, procesamiento, comercialización), como en tierras agrícolas. El objetivo de este artículo es el análisis de la reciente participación del fondo de pensiones público de Canadá (CPPIB), el octavo a nivel mundial, en la división agrícola de una de las cinco principales empresas globales alimentarias: Glencore.
\end{abstract}

Palabras clave: financiarización; empresas agro-alimentarias; fondos de pensiones; CPPIB; Glencore.

\section{THE FINANCIALIZATION OF AGRICULTURE: THE PARTICIPATION OF THE CANADA PENSION PLAN IN GLENCORE}

\section{ABSTRACT}

The financialization of the agri-food system, accelerated as a result of the crisis of 2007-08, has led to the diversification and strengthening of so-called financial agents. These include institutional investors, who group entities such as pension funds (private and public). Among the investment strategies of these have acquired relevance in the agro-food sector, both in the different stages of value chains (sourcing, production, processing, marketing), and farmland. The objective of this article is to analyze the recent participation of the public pension fund of Canada (CPPIB), the eighth worldwide, in the agricultural division of one of the five leading global food companies: Glencore.

Key words: financialization; agribusiness; pension funds; CPPIB; Glencore.

\footnotetext{
${ }^{1}$ Instituto de Geografía, Universidad Nacional Autónoma de México, Avenida Universidad 3000, Delegación Coyoacán, México DF. 04510. E-mail: flavia2451@gmail.com

${ }^{2}$ La autora agradece el financiamiento otorgado por la Universidad Nacional Autónoma de México (UNAM), proyecto PAPIIT \#IN300717.
}

Fecha de recepción: 17 de marzo de 2017. Fecha de aceptación: 16 de junio de 2017. 


\section{INTRODUCCIÓN}

En años recientes, una gama de instituciones financieras, incluidos bancos, fondos de inversión (privados y soberanos), fondos de cobertura (hedge funds) y fondos de pensión, han estado realizando grandes inversiones en alimentos y tierras alrededor del mundo, contribuyendo así a la llamada "financiarización" del sistema alimentario mundial (ISAKSON, 2014; CLAPP, 2013). Esta ha sido definida por EPSTEIN (2005) como la creciente importancia de los mercados, motivos, instituciones y élites, todos éstos de índole financiera, en la operación de la economía y sus instituciones gobernantes, tanto a nivel nacional como internacional (citado en LAWRENCE et al., 2015). De acuerdo a ISAKSON (2014), la actual fase de financiarización que se ha venido desarrollando desde fines de los 1970's ha permeado casi todos los aspectos del sistema alimentario, expandiéndose marcadamente a raíz de la crisis alimentaria, energética y financiera del 2007-08, la cual generó una mayor conciencia sobre la escasez y el carácter finito de los recursos naturales (SIPPEL et al., 2016; MARTIN y CLAPP, 2015). El proceso de financiarización ha transformado diversas esferas del sistema alimentario a nivel mundial, como es el comercio y procesamiento de alimentos (SALERNO, 2014; CLAPP, 2015; ISAKSON, 2014; BURCH y LAWRENCE, 2013); el manejo de riesgos y precios (ISAKSON, 2014), y el sector de insumos y tierra agrícola (SIPPEL et al., 2016; LARDER et al., 2015; LAWRENCE et al., 2015; ISAKSON, 2014).

Dentro de los agentes participantes del proceso de financiarización se encuentran los llamados inversores institucionales, quienes invierten el dinero de una gran cartera de clientes para comprar instrumentos financieros (bonos, opciones, contratos a futuro, acciones, etc.), bienes raíces, infraestructura y otros bienes de inversión. Dicho tipo de inversores incluyen bancos comerciales, compañías de seguros, fondos de pensión, fondos mutualistas, fondos soberanos (estatales) de inversión, fondos de coberturas y donaciones de universidades y fundaciones (INVESTOPEDIA, 2017). Dado que su objetivo es incrementar las ganancias a mediano y largo plazo, dichos agentes institucionales diversifican sus opciones de inversión, siendo cada vez más relevante la compra de tierras agrícolas y de participaciones en compañías que conforman la cadena de valor agrícola, desde las dedicadas a la producción, hasta aquellas enfocadas en la comercialización, procesamiento y distribución de alimentos. Este es el caso de los fondos de pensión públicos (administrados por los gobiernos), cuyas inversiones en agricultura se han incrementado aceleradamente en los últimos años (BUXTON et al., 2012), de tal modo que para 2016, el principal porcentaje (20\%) de las inversiones institucionales en agricultura fueron las realizadas por dichos fondos (PREQIN, 2016). Estos, al igual que otros agentes financieros, reaccionaron ante la mencionada crisis del 2007-08, buscado otras esferas de inversión a largo plazo, como son las llamadas commodities, entre las que figuran alimentos y tierras agrícolas. De acuerdo a un estudio de la FAO (2013), en los últimos años han aumentado las inversiones de dichos fondos en entidades privadas (por ejemplo, en grandes firmas agrícolas y/o de almacenamiento y logística), entidades públicas y fondos indexados (commodity index funds). Las adquisiciones de tierras agrícolas, tanto por los fondos de pensiones como por el resto de los inversores institucionales, todavía representan un reducido porcentaje dentro de sus portafolios de inversión (FAO, 2013), pero se están incrementando aceleradamente (GRAIN, 2011; 2016; FAO, 2013; MAXWELL y RITTE, 2016), por lo que profundizaremos en este tema más adelante.

Existen pocos trabajos académicos que den cuenta del desempeño de los inversores institucionales en el proceso de financiarización (véase LARDER et al., 2015), pero un gran vacío en el caso particular de los recientes vínculos e inversiones de los fondos de pensiones en el sector agro-alimentario. El presente artículo tiene como objeto contribuir a ese conocimiento, mediante el análisis de lo que ha sido uno de los eventos actuales más relevantes: la compra en 2017 por parte del octavo fondo de pensiones público más importante a nivel mundial, el CPPIB (Canada Pension Plan Investment Board), del 40\% de participación en Glencore Agriculture, la división agrícola de Glencore, una de las cinco mayores firmas globales del sector alimentario. 
La metodología utilizada se conformó por el análisis de documentos disponibles al público, ubicados en los sitios web de Glencore, del CPPIB, y de diversos inversores institucionales (fondos de pensión públicos y privados, fondos de donaciones, etc.). Dado que Glencore es una firma pública, fue posible obtener información sobre sus antecedentes históricos, la importancia y características de sus diversas esferas de actuación (productiva, financiera, servicios, etc.), los escenarios geográficos de sus actividades y sus perfiles corporativos. También se analizaron los reportes financieros generados por Glencore entre 2011 y 2017, y se acopió información sobre la firma en otros medios (revistas, videos, notas periodísticas, etc.). Para profundizar en la actividad agrícola de Glencore, se acudió a los sitios web de las empresas que tiene en diversos países, como las dedicadas a la producción de biodiesel, aceites comestibles, harinas y pastas de granos. Los hallazgos obtenidos por los medios reseñados fueron complementados con la información proporcionada en entrevista directa por el Gerente Comercial de Granos de Glencore (2016). En el caso del fondo de pensiones CPPIB, como de los otros sobre los que damos información con el objeto de proporcionar una visión más completa de ese tipo de inversores, la obtención de información fue más complicada. Pocos son los fondos que dan datos específicos sobre sus inversiones en agricultura, señalando cuando mucho el porcentaje que esta actividad representa dentro de sus activos totales. Las inversiones en actividades forestales, compra y/o renta de tierra agrícola y participación en agronegocios, están contenidas en rubros que cada fondo denomina de distinta manera ("entidades socialmente responsables", "inversiones verdes", "otras inversiones", "bienes raíces", "infraestructura", "fondos de inversión privados", etc.), siendo común que en ellos se engloben inversiones realizadas en muy distintas actividades (por ejemplo, metales y minerales, energía y agricultura), no proporcionándose información desglosada. Por ello, recurrimos a diversas fuentes de información publicadas en medios electrónicos, a las publicaciones de organismos como la FAO, GRAIN y OCDE, y a la producción académica existente. Finalmente, se llevó a cabo una revisión de literatura sobre empresas globales alimentarias, el proceso de financiarización de la agricultura y los inversores institucionales.

En el siguiente apartado se reseñarán los antecedentes e importancia de Glencore como empresa global, seguido de aquel donde se analiza el desempeño de dicha firma en el ámbito agrícola mundial. En el cuarto apartado se describe el papel jugado por la firma Viterra, pieza clave de la actividad agrícola de Glencore; en el quinto se analizan las características del CPPIB y su participación en Glencore Agriculture, proporcionándose información sobre las inversiones en agricultura de otros fondos de pensiones relevantes a nivel mundial, para terminar con las conclusiones.

\section{GLENCORE: EL IMPERIO DE UNA EMPRESA GLOBAL}

En 1974, el estadounidense Marc Rich fundó en Suiza la firma Marc Rich \& Co. AG, dedicada al comercio de metales, minerales y petróleo (crudo y derivados). Sin embargo, en 1983 Rich fue inculpado por la justicia de Estados Unidos por uno de los mayores fraudes por evasión fiscal, y por compra ilegal de petróleo a Irán, pero logró huir a Suiza y, años después, fue perdonado por el presidente Bill Clinton el último día de su mandato (BERG, 1983). A partir de 1987, Marc Rich \& Co. inició un proceso de diversificación más allá de la esfera comercial, mediante adquisiciones en el ramo de la minería, fundición, refinamiento y procesamiento (GLENCORE, 2017). En 1990 adquirió una participación en Xstrata, una de las principales firmas productoras de carbón a nivel mundial y el mayor exportador de carbón térmico, cobre, níquel, vanadio primario y zinc (RISEBOROUGH y BLAS, 2015), todo lo cual posicionó a Marc Rich \& Co. entre las firmas líderes globales. Pero en 1994, después de fallar en el control del mercado del zinc, Rich fue forzado por el resto de los accionistas a vender el $51 \%$ de las acciones que tenía en la firma, a la cual los nuevos directores denominaron Glencore International (GI) (acrónimo de Global Energy Commodity Resources). Esta empresa se hace pública en 2011, al enlistarse en la Bolsa de Valores 
de Londres, ubicándose por su valor (cerca de 60 billones de dólares), por arriba de Boeing y Ford Motor Company, y posicionando al que hasta hoy es su CEO, Iván Glasenberg, como uno de los hombres más ricos de Europa (SILVERSTEIN, 2012).

En 2013, GI completa su fusión con Xstrata, y pasa a llamarse Glencore PLC, conservando su sede en Baar, Suiza (GLENCORE, 2017). Ya para entonces, dicha firma (a la que nos referiremos como Glencore), ocupaba el segundo lugar entre las diez mayores comercializadoras de mercancías a nivel global, antecedida por Vitol, el principal comerciante de petróleo y gas del mundo. El tercer sitio lo ocupaba Cargill, seguida por Trafigura, Koch Industries, Mercuria Energy, Noble Group, Gunvor Group, Archer Daniels Midland (ADM) y Bunge (SZALA, 2013). Siete de las mencionadas firmas están mayoritariamente en el ramo de energía y/o metales, aunque cinco de ellas participan también en la producción y/o comercio de productos agrícolas, como es el caso de Glencore PLC. Para 2014, esta empresa ocupó el décimo lugar entre las 500 mayores empresas globales; sin embargo, y por problemas financieros que reseñaremos posteriormente, en 2015 descendió hasta el catorceavo sitio, reportando un ingreso anual de 170.5 billones de USD (FORTUNE, 2017).

Glencore PLC tiene tres segmentos centrales de actividad: metales y minerales, productos de energía y productos agrícolas, participando a nivel mundial en la producción, procesamiento, refinación, transporte, almacenamiento, financiamiento y abasto de los mencionados bienes (GLENCORE, 2017). Es una de las compañías globales más importantes de recursos naturales; en metales y minerales es muy relevante en cobre, zinc, aluminio, níquel, aleaciones de fierro, hierro y cobalto. En energía está principalmente en carbón y petróleo y, en agricultura, en granos (trigo, cebada, maíz y arroz), oleaginosas, aceites comestibles, biocombustibles, algodón y azúcar (GLENCORE, 2015, 2017). En 2012, Glencore controlaba poco más de la mitad del comercio global de zinc y cobre, y cerca de una tercera parte del carbón transportado por mar, manejando además el 3\% del consumo diario global de petróleo. De éste, abastecía desde firmas estatales de energía en Brasil e India, hasta multinacionales como Exxon Mobil y Chevron. En la rama agrícola ya era uno de los mayores exportadores de granos, participando con casi el 10\% del mercado global (SILVERSTEIN, 2012; SZALA, 2013). Glencore señala que los bienes físicos que comercia y distribuye provienen tanto de su propia producción como de terceros, abasteciendo a consumidores industriales como los del sector de alimentos y procesadores de aceites, industria automotriz, acerera y generadora de energía. También otorga a productores y consumidores servicios financieros, de manejo de riesgos y de logística (GLENCORE, 2017).

En 2015, Glencore intervenía en la producción y manejo de alrededor de 90 mercancías o commodities, para lo cual tenía presencia en más de 50 países, empleando a 160,000 personas. Tuvo ingresos brutos (revenues) por 172.7 billones de USD. De éstos, el 49\% (83.6 billones USD) procedió de su sector de energía; 38\% (66 billones USD) de metales y minerales, y 13\% (23 billones USD) de la agricultura. La procedencia geográfica de sus ingresos fue la siguiente: $38 \%$ de Asia, 32\% de Europa, 19\% de América, 7\% de Oceanía y 4\% de África (GLENCORE, 2017, 2016a). En metales y minerales, Glencore señala que mientras los mineros tradicionales (BHP, Río Tinto, Vale, AngloAmerican) exploran, producen, procesan y refinan, y los comerciantes (Noble Group, Cargill, Bunge, Trafigura y Vitol) están en logística y comercialización, Glencore cubre exploración, producción, procesamiento/refinamiento, logística y comercialización, siendo esta integración a lo largo de la cadena lo que le otorga a la firma fuerza competitiva (GLENCORE, 2017). De hecho, en 2015 Glencore ocupó el sexto sitio dentro de las mayores empresas mineras globales (PRICEWATERHOUSECOOPERS, 2016). Para su desempeño, Glencore cuenta con 150 facilidades (mineras y metalúrgicas, petróleo y agricultura), valuadas en 2015 en 128 billones de USD. Su red de instalaciones en producción, almacenaje, manejo, procesamiento y facilidades portuarias se ubica en más de 35 países de todos los continentes (GLENCORE, 2016a). 


\section{GLENCORE, UN GIGANTE DE LA AGRICULTURA MUNDIAL}

Glencore incursiona en el sector agrícola en 1981, cuando Marc Rich adquiere Granaria Group, una comercializadora holandesa de granos. Actualmente, la mayoría de los ingresos de la actividad agrícola de Glencore proceden del comercio de bienes agrícolas, el cual generó el $91 \%$ de dichos ingresos en 2015, mientras que el restante porcentaje procedió de sus actividades industriales y de producción agrícola (farming). En ese año, comercializó 43.7 millones de toneladas (MT) de granos, 23.3 MT de oleaginosas y aceites, 0.4 MT de algodón y 1.1 MT de azúcar (GLENCORE, 2016a). La mayor parte de los bienes producto de sus actividades industriales procedieron de la molienda de oleaginosas (aceites y otros), seguida por el procesamiento de caña de azúcar, molienda de trigo y arroz, biodiesel y producción agrícola.

Glencore participa a nivel global en la producción, manejo, almacenamiento, procesamiento y comercialización de productos agrícolas. Comercializa granos (trigo, maíz, cebada), oleaginosas y leguminosas, así como diversos productos procesados (aceites comestibles, azúcar, algodón, arroz, harina de trigo, alimentos animales y biodiesel). Con operaciones en más de 35 países, Glencore se ubica entre los tres principales exportadores de granos y oleaginosas de Rusia, la Unión Europea, Canadá y Australia, países claves en la producción y comercio global de granos, y entre los tres mayores comercializadores globales (vía marítima) de trigo, cebada forrajera, leguminosas secas (frijol, lenteja y chícharo), canola y girasol (semilla, aceites y pasta). En 2015, la parte agrícola de la firma, Glencore Agriculture, comercializó 68.5 millones de toneladas de productos agrícolas, de las cuales el 64\% (43.7 millones de toneladas) fueron granos (GLENCORE, 2017).

Glencore cuenta con 274 facilidades para almacenamiento y manejo de granos ubicadas en 17 países, entre las que sobresalen por su nivel las de Canadá, Australia, Argentina, Rusia y Ucrania; 36 facilidades para procesamiento y refinamiento (12 países); 23 terminales portuarias ( 8 países) y 96,000 hectáreas de tierra cultivada (3 países) (GLENCORE AGRICULTURE, 2017a) (Figura 1). Sus plantas de trituración de oleaginosas (soja, girasol, canola y colza) se ubican en 9 países (Argentina, Brasil, Hungría, República Checa, Ucrania, Alemania, Polonia, Canadá y China). En Argentina participa en la que es la mayor planta moledora de soja de ese país (Renova Timbúes), dedicada a la producción de aceite, harina de soja y lecitina, así como en la mayor firma productora de biodiesel (Renova San Lorenzo). Posee también una planta productora de aceite y pasta de girasol (Moreno Group) que figura entre las mayores productoras y exportadoras mundiales. En Brasil tiene 6 molinos de trigo, uno de oleaginosas y otro de caña de azúcar para la fabricación de azúcar y etanol y, en Argentina, Brasil y Uruguay cuenta con 4 molinos de arroz (GLENCORE, 2014a). En Europa sobresalen sus plantas extractoras de aceite de oleaginosas (República Checa, Polonia, Hungría, etc.) y productoras de biodiesel (Alemania, Holanda y Suiza), además de infraestructura de almacenamiento y terminales portuarias (GLENCORE, 2014b, 2017). En Canadá y Australia cuenta con importantes facilidades de almacenamiento de granos (82 y 6, respectivamente), además de 11 puertos, mayoritariamente para las actividades de su firma Viterra.

La dimensión de Glencore es tal, que hoy día es obligado incluir a esta firma entre las cinco mayores que controlan el comercio mundial de granos, oleaginosas y azúcar, integrándose el grupo por ADM (Archer Daniels Midland), Bunge, Cargill, Glencore y Dreyfus. La diversificación de actividades más allá del sector agro-alimentario es una característica común: Glencore, Cargill y Dreyfus están además en energía y/o metales; Cargill, por ejemplo, es importante en el comercio de petróleo crudo, gas, gasolina y acero, mientras que Dreyfus es uno de los cinco principales comerciantes a nivel global de cobre y zinc, con presencia en las principales regiones de producción y venta de dichos metales (DREYFUS, 2016). Cargill, Bunge y Dreyfus son relevantes también en biocombustibles, a lo que Bunge añade además el comercio de fertilizantes (SZALA, 2013). 


\section{LA IMPORTANCIA DE VITERRA}

La firma Viterra ocupa una posición clave en el comercio de granos de dos países que figuran entre los cinco principales exportadores de esos bienes a nivel mundial: Canadá y Australia. El primer país ocupó en 2015/2016 el tercer lugar a nivel mundial como exportador de trigo, mientras que Australia fue el segundo mayor exportador de cebada (USDA, 2017). Viterra surgió en Canadá en 2007, habiendo sido sus predecesores cooperativas comercializadoras (wheat pools) establecidas durante los años 1920's, entre las que resalta la Saskatchewan Wheat Pool. La ola privatizadora de las cooperativas de productores y el retiro del Estado del control del comercio de granos, iniciado en Canadá y reproducido en Australia, fueron escenarios idóneos para la expansión de Viterra, quien en 2009 compra ABB Grain Ltd, compañía privada cuyo origen fue el Consejo Australiano de la Cebada. Viterra se convierte en el principal manejador de granos del oeste de Canadá y de Australia del Sur, operando también en países como Estados Unidos, Nueva Zelanda y China, con oficinas en Japón, Singapur, Vietnam, Suiza, Italia, Ucrania, Alemania, España e India. Operaba tres segmentos: manejo y comercio de granos, agro-productos y procesamiento (VITERRA, 2012). Sin embargo, a fines de 2012, Viterra es comprada por Glencore, con el fin de acceder al mercado de granos de Canadá y reforzarse en el de Australia, donde tenía presencia desde 2003 a través de Glencore Grain (GLENCORE, 2013). A raíz de dicha adquisición, Viterra (sigue conservando este nombre), expande aún más sus actividades, figurando entre los líderes exportadores de granos de Australia, donde acopia, maneja, almacena, transporta y comercializa diversos bienes (trigo, cebada, oleaginosas, leguminosas, alimentos animales y algodón), apoyada por una amplia red de infraestructura de almacenamiento y modernas facilidades portuarias (VITERRA, 2016a, 2016b, 2017). El principal espacio de actuación de Viterra es Australia del Sur, donde la firma comercializa un $45-55 \%$ del grano producido en esta región, lo que, aunado a sus ventas de grano de la parte Oeste de Australia, da como resultado el control del 35\% de todo el grano producido en ese país, muy por arriba de la firma Cargill (15\%) (LYNDON, 2016).

FIGURA 1

Operaciones globales de Glencore en el sector agro-alimentario

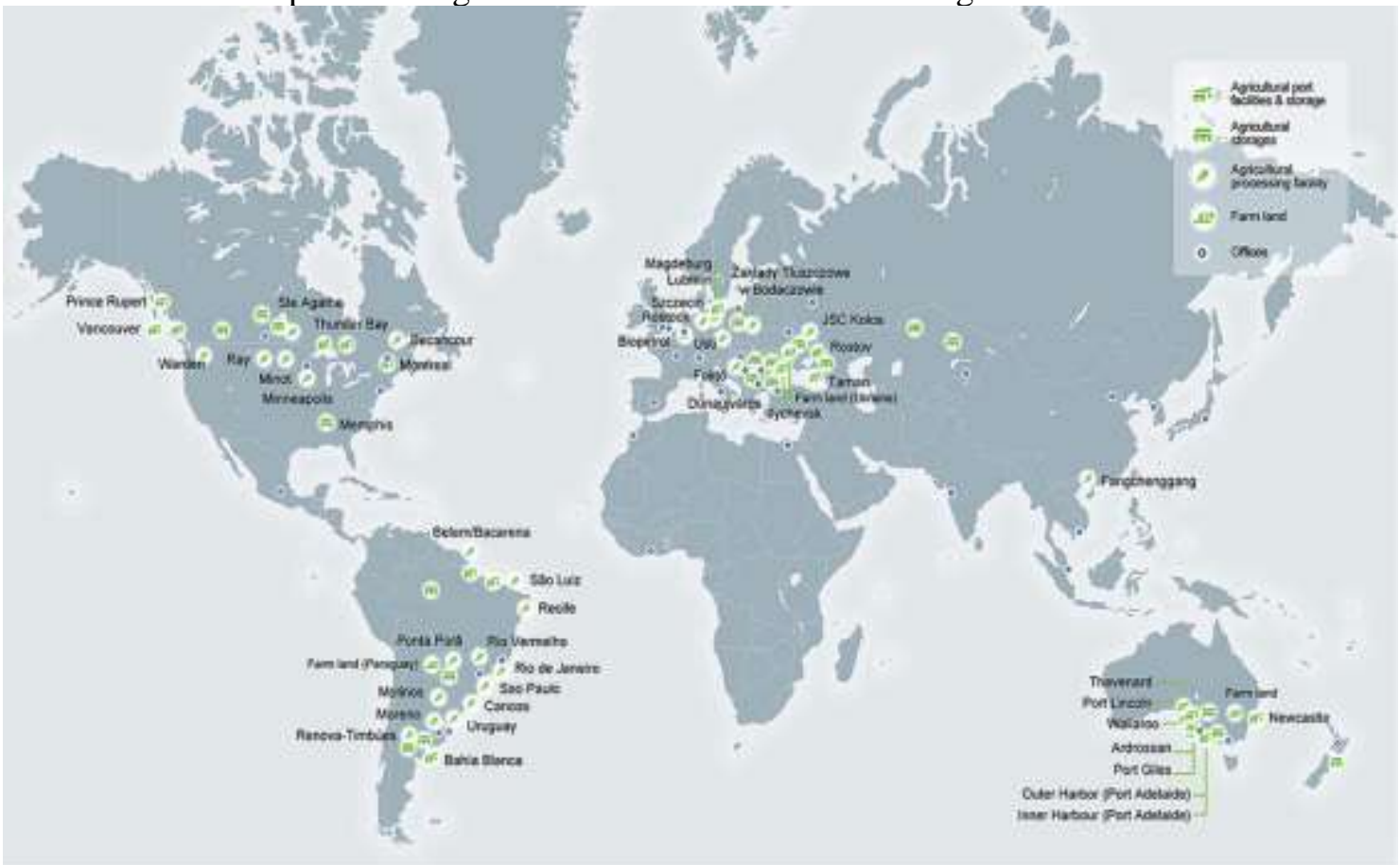

Fuente: Glencore Agriculture, 2017b. 
Viterra mantiene sus oficinas centrales en Regina (Saskatchewan, Canadá), y entre sus operaciones industriales en ese país destaca la planta Becancour (Quebec), la mayor procesadora de oleaginosas del este de Canadá, que produce aceite comestible, pasta para alimentos animales y aceite para la elaboración de biodiesel. Sus productos son distribuidos en Norteamérica, Europa y Sudamérica. La otra fábrica digna de mención es St. Agathe (en Manitoba), donde se produce aceite comestible y pasta de canola, ambos no genéticamente modificados, y que constituye una de las mayores procesadoras a nivel mundial de este tipo de bienes. Para esto, así como para la comercialización de granos y leguminosas, Viterra cuenta con 6 facilidades portuarias desde donde envía a 50 países (VITERRA, 2017).

\section{EL FONDO DE PENSIONES DE CANADÁ Y GLENCORE AGRICULTURE}

Durante los años previos a 2016, Glencore venía siendo afectado por el descenso de los precios de ciertos minerales (cobre, hierro, zinc, carbón, aluminio y níquel), a lo que se sumó en 2015 el declive en el crecimiento de China, el descenso de los precios del petróleo y la fortaleza del dólar estadounidense. Sus ingresos brutos en ese año decrecieron en un $30 \%$ con respecto a 2014, además de que la firma venía presentando una deuda considerable, que aunque se había reducido en los últimos años, en 2015 ascendió a 25.9 billones de USD. Glencore se planteó reducir esta deuda para fines de 2016, tratando de conservar su calificación crediticia, para lo cual decidieron reducir proyectos y vender algunas propiedades. Así, se puso en venta un 50\% de las participaciones en su negocio agrícola (Glencore Agriculture), al igual que algunas minas e infraestructura ferroviaria en Australia (GLENCORE, 2016a, 2016b). En su división agrícola, los ingresos brutos por agricultura se habían reducido en 2015, debido a la normalización de la cosecha de granos en Canadá (después de una producción récord en 2014); la imposición de Rusia de un impuesto a la importación de trigo; un entorno de menores precios de las commodities; cambios en la oferta y demanda, y fluctuaciones en los tipos de cambio. Para fines de 2016, los ingresos brutos por agricultura volvieron a descender (de 23.1 a 20.6 billones de USD) debido a menores cosechas y calidad de los granos en Canadá y Australia. En ese año, Glencore Agriculture se valuaba en 6.25 billones de USD (GLENCORE, 2016a, 2016b, 2017).

El 1 de diciembre de 2016, Glencore concluyó la venta del 50\% de participación en Glencore Agriculture por un total de 3.1 billones de USD. Los compradores fueron el CPPIB (Canada Pension Plan Investment Board) y la British Columbia Investment Management Corporation (BCIMC), quienes adquirieron el $40 \%$ y $10 \%$ de participación, respectivamente. Marc Jenkins, uno de los directivos del CPPIB, al referirse a la mencionada compra, señaló que la agricultura era un excelente inversión de largo plazo para dicho fondo de pensiones, y que su participación en Glencore Agriculture complementaba su portafolio existente de propiedades en este sector de actividad, otorgándole una mayor cobertura global y diversificación, y una reducción de riesgos geográficos (GLENCORE, 2017). Los antecedentes de las inversiones agrícolas del CPPIB se remontan a 2012, cuando este fondo empezó a comprar tierras en Norteamérica y, desde entonces, ha adquirido casi 100,000 hectáreas en Estados Unidos y Canadá. Parte de estas tierras las conforman las 48,500 hectáreas compradas en 2013 en Saskatchewan, Canadá, a Assinoboia Farmland LP, por 95 millones de USD. El fondo tenía intención de expandir sus compras de tierras en dicha provincia, pero la presión de los productores logró que el gobierno prohibiera a algunos inversores institucionales realizar este tipo de adquisiciones, truncando los planes del CPPIB. Este continuó adquiriendo tierras en Estados Unidos, pero suspendió sus planes de compras en Australia, Nueva Zelanda y Brasil, manifestando recientemente que no intentaba adquirir más tierras agrícolas y que incluso deseaba vender las existentes en su portafolio, para concentrarse en el procesamiento, almacenaje y comercialización de productos agrícolas, a raíz de su participación en Glencore (TILAK y SCUFFHAM, 2017).

El CPPIB, fundado en 1997, constituye el más importante fondo público de pensiones de Canadá (BÉDARD-PAGÉ et al., 2016), dedicado a invertir los fondos sobrantes del Plan de Pensiones de 
Canadá (CPP), una vez que éste ha pagado las pensiones de alrededor de 20 millones de contribuidores o beneficiarios. Dicho Plan invierte en bienes públicos, privados, bonos, deuda privada, sector inmobiliario, infraestructura, agricultura, recursos naturales y otras áreas de inversión, teniendo su sede en Toronto, Canadá, y oficinas en diversos países (Hong Kong, Londres, Luxemburgo, Mumbai, Nueva York y San Pablo (Brasil). En 2015, el CPPIB ocupó el octavo lugar entre los mayores fondos de pensiones públicos del mundo (Tabla 1) y, para principios de 2017, el valor de sus bienes fue de 281 billones de USD (PENSION\&INVESTMENTS, 2017; PREQIN, 2017).

TABLA 1

Los diez mayores fondos de pensión públicos a nivel mundial (2015)

\begin{tabular}{|l|r|r|}
\hline \multicolumn{1}{|c|}{ Nombre } & País & $\begin{array}{c}\text { Valor de } \\
\text { los activos } \\
\text { (Millones } \\
\text { de USD) }\end{array}$ \\
\hline Government Pension Investment Fund (GPIF) & Nopón & $1,143,838$ \\
\hline Government Pension Fund Global & Corea del Sur & 829,031 \\
\hline National Pension Service (NPS) & USA & 422,200 \\
\hline Federal Retirement Thrift Investment Board's (FRTIB) & Países Bajos & 418,745 \\
\hline APG (All Pensions Group) & USA & 296,744 \\
\hline California Public Employees (CalPERS) & China & 247,361 \\
\hline National Social Security & Canadá & 228,431 \\
\hline Canada Pension Plan Investment Board (CPPIB) & Países Bajos & 215,006 \\
\hline PFZW (Pension Fund for Care Netherlands and Well-Beingy) & Singapur & 207,872 \\
\hline Central Provident Fund (CPF) & & \\
\hline
\end{tabular}

Fuente: Pensions\&Investments, 2017.

El CPPIB ha expandido geográficamente sus inversiones, de tal modo que a principios de 2017, el $81 \%$ de su portafolio se ubicaba fuera de Canadá, con el objetivo de diversificar riesgos y aprovechar las oportunidades del mercado global. Reportaba actividades en Estados Unidos, Europa, Reino Unido, Canadá, Asia, América Latina y Australia. En Norteamérica posee Antares Capital, prestamista líder en Estados Unidos, participando también en infraestructura (carretera, energía), software, seguros y sector inmobiliario; en Europa invierte en comercio detallista, sector inmobiliario, logística, desarrollo industrial y entretenimiento, y en Asia tiene inversiones en el principal banco de China, en el sector de grandes superficies (supermercados) (Corea del Sur), y en desarrollo de logística (China) (CPPIB, 2016). Es claro que la reciente participación del CPPIB en Glencore Agriculture expandirá de manera relevante el espacio geográfico de sus inversiones y la importancia de éstas, pero datos concretos se tendrán hasta la presentación de informes de dicho fondo en 2017.

El otro nuevo participante en Glencore Agriculture, el comprador minoritario (BCIMC) es el sexto mayor fondo público de pensiones de Canadá. Con un portafolio global de más de 127 billones de dólares canadienses, dicho fondo de pensiones otorga beneficios a 538,000 miembros, así como a los fondos de seguros y beneficios de 2.3 millones de personas en Columbia Británica. $\mathrm{Al}$ igual que el otro fondo reseñado, la BCIMC realiza inversiones en toda una gama de sectores: ingresos fijos, hipotecas, sector inmobiliario, infraestructura, recursos renovables y firmas manejadoras de inversiones (públicas y privadas) (BCIMC, 2016; BÉDARD-PAGÉ et al.; 2016, NAIR, et al.; 2016).

Los casos anteriormente mencionados forman parte de un proceso relativamente nuevo, en el que fondos de pensiones (privados y públicos) y fondos de donaciones (endowments funds) han 
incrementado sus inversiones en el sector agrícola y alimentario, como parte de una estrategia alternativa de asignación de recursos, sobre todo en el caso de los fondos de Estados Unidos, Canadá y Europa, pero también de Australia y Sudáfrica (UNEPFI, 2012). Las ganancias que dichos inversionistas están obteniendo de la agricultura superan con mucho a las obtenidas por otros instrumentos de inversión (bonos del tesoro, stocks, etc.), además de que, aquellos que han invertido en tierras agrícolas, cuentan con la creciente apreciación del valor de este activo (FAO, 2013). Los fondos estadounidenses han sido pioneros en este tipo de inversiones, pero recientemente los fondos europeos y de otros países se han sumado a dicha tendencia; por ejemplo, en 2016, el UK Environment Agency Active Pension Fund se planteó dirigir hasta un 4\% de sus inversiones totales hacia tierras agrícolas y forestales (ENVIRONMENT AGENCY, 2016), mientras que el New Zealand Superannuation Fund está invirtiendo el 7\% de sus activos en tierras forestales (producción de madera) y agrícolas (NZSF, 2017). Por su parte, el APG (All Pensions Group), un relevante fondo de pensiones de los Países Bajos, reportó en 2013 que $0.25 \%$ de sus activos totales estaban invertidos en tierra agrícola en Australia, Europa del Este, India y América Latina, dado que consideraban que estas inversiones eran menos volátiles y otorgaban ganancias del 8-12\%, y hasta del 20\% (FAO, 2013), mientras que el fondo de pensiones PKA (Dinamarca), detenta tierras en África, para producir diversos bienes (cereales, soja, frutas, vegetales, azúcar, té y café). Ejemplos de otros fondos con inversiones en tierras agrícolas (algunos incluidos entre los diez mayores fondos públicos de la Tabla 1) son el PFZW (Países Bajos), AP2 (Suecia) (segundo en importancia en su país y uno de los mayores fondos de pensiones europeos), BT Pension Scheme, Railpen (UK), Pension Protection Fund (UK), CalPERS (California Public Employee's Retirement System) (USA), Harvard University Endowment Fund (USA) y TIAA-CREF (USA) (para un listado más amplio, véase GRAIN, 2012). El mencionado PFZW (Pension Fund for Care Netherlands and Well-Beingy) tenía en 2012 invertido en tierras agrícolas el 0.3\% de su portafolio de inversiones. En 2010 invirtió 50-100 millones de euros en Black River Asset Management (fondo de inversiones de Cargill dedicado a la compra de tierras), además de producir diversos bienes agrícolas en Asia, destinados mayoritariamente al mercado chino. El PFZW también ha invertido en NCH Capital (con sede en Nueva York), para la compra y/o renta de tierras en Rusia y Ukrania, además de tener participación en Adecoagro, firma que posee 300,000 hectáreas de tierras en Argentina, Brasil y Uruguay (GRAIN, 2012).

Por su parte, el fondo de pensiones CalPERS mantiene una relevante inversión en Black Earth Farming, además de tener participación en relevantes firmas de agro-negocios (Olam, Indofood, Wilmar, Sime Darby, Golden Agri-Resources y IOI Corp), que hacen producir tierras en África, Asia y América Latina. (GRAIN, 2012). Entre los fondos de inversión privados, destaca el mencionado TIAA-CREF (USA), el más relevante de Estados Unidos y uno de los mayores a nivel mundial. Este fondo, que agrupa a 5 millones de participantes del sector académico, médico y de investigación de ese país, ha creado dentro de su estructura entidades dedicadas a la inversión en tierras agrícolas, disponiendo en 2014 de 566,560 hectáreas (valuadas en 5.1 billones de USD), ubicadas en Brasil, Australia y Estados Unidos, para la producción de granos (cebada, maíz, trigo, soja y leguminosas), oleginosas, azúcar, uvas, frutas y vegetales (TIAA-CREF, 2015). Desde 2012, TIAA-CREF estableció el Global Agriculture LLC, teniendo como co-inversores al fondo de pensiones sueco AP2, el British Columbia Investment Management Corporation (IMC) y la Caisse de dépôt et placement du Quebec. Para 2016, el fondo había invertido 8 billones de USD en agricultura (MAXWELL \& RITTE, 2016).

Como ya mencionamos, uno de los destinos favoritos de los inversores institucionales ha sido Australia, donde relevantes administradores de fondos globales y algunos de los mayores fondos de pensiones a nivel mundial han comprado importantes cantidades de tierras agrícolas, invirtiendo más de 1.5 billones de USD durante 2010-13. Estos inversores incluyen dos fondos de pensiones suecos (Första AP y AP2), el alemán Algemene Pensioen Groep; el danés Danske; el suizo Adveq Real Assets Harvested Resources; el fondo soberano de Qatar's, el TIAA-CREF, y otros de Canadá (British Columbia Investment Management Corporation, BNY Mellon, Ontario Municipal 
Employee Retirement System, Quebec's CDPQ, y la Caisse de dépôt et placement du Quebec) (CRANSTON, 2013). El fondo Första AP, por ejemplo, a través de una subsidiaria (First Australian Farmland Fund) tiene inversiones en Australia y Nueva Zelanda, contando en el primer país con 16,000 hectáreas, mientras que el fondo estadounidense TIAA-CREF, mediante su firma subsidiaria dedicada a inversiones globales en tierras (Westchester Agricultural Assets Management), posee 26,000 hectáreas en Australia (MAGNAN, 2015). A estas inversiones habría que añadir las realizadas por los propios fondos de pensiones australianos.

\section{CONCLUSIONES}

La financiarización del sistema global alimentario es una de las transformaciones más significativas que el mundo está presenciando (LAWRENCE et al., 2015), constituyendo un fenómeno complejo que ha dado lugar a un mayor uso de ciertos instrumentos financieros (ej. mercados de futuros), así como a la aparición, tanto de otro tipo de instrumentos, como de nuevos agentes financieros. Esencialmente, la financiarización ha implicado una nueva configuración de las relaciones entre el sector productivo y el financiero, para cuyo entendimiento resulta indispensable el estudio de los sujetos que se encuentran detrás de dicho proceso (GARZÓN, 2009). Entre los nuevos agentes financieros se encuentran los llamados inversores institucionales, dentro de los cuales los fondos de pensiones (públicos y privados) están siendo los más relevantes en cuanto a inversiones en agricultura, las cuales se han expandido en años recientes más allá del rubro de tierras agrícolas, incluyendo además inversiones en empresas de capital privado (private equity funds) (ej. grandes compañías agrícolas y firmas de almacenamiento y logística), fondos públicos y fondos de productos indexados (commodity index funds). En el caso de Estados Unidos, los fideicomisos para la inversión en bienes inmuebles son instrumentos muy comunes para invertir en tierras agrícolas (FAO, 2013).

De acuerdo a la FAO (2013), los inversores globales en agricultura pueden ser clasificados en tres categorías: 1) los que ubican a la agricultura como una inversión en bienes raíces, esperando ganancias de la renta y apreciación de las tierras, sin participar activamente en la administración de las granjas, 2) los que se enfocan activamente en la administración de las operaciones, buscando ganancias, tanto por esta actividad, como por la apreciación de las tierras, y 3) los que invierten en agricultura como una fuente de materias primas para actividades agroindustriales (FAO, 2013). Los diversos ejemplos dados en este artículo sobre relevantes fondos de pensiones que están invirtiendo en agricultura demuestran que la mayoría de ellos se ha concentrado en las dos primeras categorías, mientras que el más importante fondo público de pensiones de Canadá, el CPPIB, ha invertido mayoritariamente en la tercera de ellas. La reciente adquisición de este fondo del $40 \%$ de participación en la división agrícola de Glencore, una de las cinco mayores firmas alimentarias a nivel mundial, es probablemente la inversión más importante en agricultura por parte de los denominados inversores institucionales. Su participación en Glencore ha convertido al CPPIB en un relevante protagonista del sistema agro-alimentario mundial, participando en diversas fases de la cadena de valor (producción, almacenaje, procesamiento, comercio, transporte, financiamiento, etc.).

Será necesario dar seguimiento a las complejas implicaciones que está teniendo el proceso de financiarización, sin embargo, y como señala ISAKSON (2014), hoy día es evidente que dicho proceso ha consolidado el poder y la riqueza de élites financieras, a expensas de los productores y trabajadores agrícolas, y exacerbado la fragilidad del sistema alimentario mundial. El fondo de pensiones analizado en este artículo (CPPIB) es ya un nuevo actor dentro de este escenario. 


\section{BIBLIOGRAFÍA}

BÉDARD-PAGÉ, G; DEMERS, A; TUER, E. y TREMBLAY, M. (2016): "Large Canadian Public Pension Funds: A Financial System Perspective", Bank of Canada. Disponible en: http://www.bankofcanada.ca/wp-content/uploads/2016/06/fsr-june2016-bedard-page.pdf [consulta: 10 de mayo de 2017].

BERG, ERIC N. (1983): The New York Times, "Marc Rich indicted in vast tax evasión case", 20 de septiembre.

BCIMC (British Columbia Investment Management Corporation) (2016): “Annual Report 201516". Disponible en: https://www.bcimc.com/Default.asp [consulta: 26 de febrero de 2017].

BURCH, D. y LAWRENCE, G. (2013): Agriculture and Human Values. "Financialization in agrifood supply chains: private equity and the transformation of the retail sector", $n^{\circ} 30, \mathrm{p}$. 247-258.

BUXTON, A., CAMPANELE, M. Y COTULA, L. (2012): "Farms and funds: investment funds in the global land rush", Disponible en: Disponible en: http://pubs.iied.org/pdfs/17121IIED.pdf [consulta: 1 de marzo de 2017].

CLAPP, JENIFFER (2013): "Financialization, Distance and Global Food Politics". Disponible en: https://www.iss.nl/fileadmin/ASSETS/iss/Research_and_projects/Research_networks/ICAS/24_ Claeys_2013-1.pdf [consulta: 3 de marzo de 2017].

CLAPP, JENIFFER (2015): Canadian Food Studies, "ABCD and beyond: From grain merchants to agricultural value chain managers", vol. 2, n 2, p. 126-135.

CPPIB (CANADA PENSION PLAN INVESTMENT BOARD) (2016): "2016 Annual Report". Disponible en: http://viewer.zmags.com/publication/cd02813c\#/cd02813c/1 [consulta: $10 \mathrm{de}$ marzo de 2017].

CRANSTON, MATHEW (2013): "Global Funds Chase Australian Farms". Disponible en: http://www.farmweekly.com.au/news/agriculture/property/general-news/global-funds-chaseaustralian-farms/2678808.aspx [consulta: 8 de mayo de 2017].

DREYFUS LOUIS COMPANY (2016): “At a glance”. Disponible en: http://ldcom.com/global/en/ [consulta: 16 de septiembre de 2016].

ENVIRONMENT AGENCY ACTIVE PENSION FUND (2016): “Annual Report and Financial Statements 2015/16". Disponible en: www.eapf.org.uk/ /media/document-libraries/[consulta: 29 de abril de 2017].

EPSTEIN, GERALD A. (2005): Financialization and the world economy. "Introduction: financialization and the world economy". En Epstein G. (ed.). Edward Elgar Publishing Limited, Cheltenham, UK, pp. 3-16.

FAO (ORGANIZACIÓN PARA LA AGRICULTURA Y LA ALIMENTACIÓN) (2013): "Emerging investment trends in primary agriculture, a review of equity funds and other foreignled investments in the CEE and CIS región". Disponible en: http://www.fao.org/3/a-i3474e.pdf [consulta: 1 de marzo de 2017].

FORTUNE (2017): The Fortune Global 500. Disponible en: http://fortune.com/global500/ [consulta: 12 de febrero de 2017].

GARZÓN, ALBERTO (2009): “Qué es la financiarización?”. Disponible en: http://agarzon.net/iqué-es-la-financiarización/ [consulta: 24 de enero de 2017].

GLENCORE (2013): "2013 Annual Report”. Disponible en: http://www.glencore.com/[consulta: 24 de febrero de 2017].

GLENCORE (2014a): “2014 Annual Report”. Disponible en: http://www.glencore.com/ [consulta: 24 de febrero de 2017].

GLENCORE (2014b): "Investor Day Presentation". Disponible en: http://www.glencore.com/ [consulta: 24 de febrero de 2017].

GLENCORE (2015): “2015 Annual Report”. Disponible en: http://www.glencore.com/ [consulta: 12 de noviembre de 2016]. 
GLENCORE (2016a): “2015 Annual Strategic Report”. Disponible en: http://www.glencore.com/assets/investors/doc/reports_and_results/2015/ [consulta: 18 de febrero de 2017].

GLENCORE (2016b): “2016 Global Metals, Mining \& Steel Conference”. Disponible en: http://www.glencore.com/assets/media/doc/speeches and presentations/2016/2016 GlobalMetals-Mining-Steel-Conference.pdf [consulta: 9 de marzo de 2017].

GLENCORE (2017): “About Us". Disponible en: http://www.glencore.com/about-us [consulta: 20 de febrero de 2017].

GLENCORE AGRICULTURE (2017a): "At a glance". Disponible en: www.glencoreagriculture.com [consulta: 20 de febrero de 2017].

GLENCORE AGRICULTURE (2017b): "Where we operate". Disponible en: www.glencoreagriculture.com [consulta: 20 de febrero de 2017].

GRAIN (2011): "Pension funds: key players in the global farmland grab". Disponible en: https://www.grain.org/article/entries/ [consulta: 28 de febrero de 2017].

GRAIN (2012): "Pension funds involved in global largescale farmland aquisitions". Disponible en: www.grain.org/attachments/2519/download [consulta: 7 de mayo de 2017].

GRAIN (2016): “The global farmland grab in 2016. How big, how bad?". Disponible en: https://www.grain.org/article/ [consulta: 28 de febrero de 2017].

INVESTOPEDIA (2017): "These investors are dominating Private Equity". Disponible en: http://www.institutionalinvestor.com/article/3545092/investors-sovereign-wealth-funds/ [consulta: 4 de marzo de 2017].

ISAKSON, S. RYAN (2014): The Journal of Peasant Studies. "Food and finance: the financial transformation of agro-food supply chains", vol. 41, n ${ }^{\circ} 5$, p. 749-775.

LARDER, N; SIPPEL, S.R. y LAWRENCE, G. (2015): Journal of Agrarian Change. "Finance Capital, Food Security Narratives and Australian Agricultural Land", vol. 15, n 4, p. 592-603.

LAWRENCE, G; SIPPEL, S. y BURCH, D. (2015): Handbook on the Globalisation of Agriculture. "The financialization of food and farming". En Robinson, G. y Carson, D.A. (eds.). Edward Elgar Publishing Limited, Cheltenham, UK, pp. 309-327.

LYNDON, ASSER (2016): Gerente Comercial de Granos de Glencore. Entrevista directa, 12 de febrero de 2016.

MAGNAN, ANDRÉ (2015): Journal of Rural Studies. "The financialization of agri-food in Canada and Australia: Corporate farmland and farm ownership in the grains and oilseed sector", $\mathrm{n}^{\circ} 41, \mathrm{p}$. $1-12$.

MARTIN, S. y CLAPP, S. (2015): Journal of Agrarian Change. "Finance for agriculture or agriculture for finance?", $\mathrm{n}^{\mathrm{0}} 15, \mathrm{p} .549-559$.

MAXWELL LOCKE \& RITTE (2016): "Pension funds investing more often in agriculture". Disponible en: http://www.mlrpc.com [consulta: 28 de febrero de 2017].

NAIR, D; BLAS, J. y CAMPBELL, M. (2016): Bloomberg. "Glencore Sells Agri Stake to Canadian Fund for $\$ 625$ Million", 8 de junio.

NZSF (NEW ZEALAND SUPERANNUATION FUND) (2017). Disponible en: https://www.nzsuperfund.co.nz/investing-nz/case-studies [consulta: 2 de mayo de 2017].

OCDE (ORGANIZACIÓN PARA LA COOPERACIÓN Y EL DESARROLLO ECONÓMICOS) (2015): "Annual Survey of Large Pension Funds and Public Pension Reserve Funds. Report on Pension's Funds' Long-Term Invetments". Disponible en: http://www.oecd.org/daf/fin/privatepensions/2015-Large-Pension-Funds-Survey.pdf [consulta: 29 de abril de 2017].

PENSION\&INVESTMENTS (2017): “The largest retirement funds". Disponible en: www.pionline.com [consulta: 28 de febrero de 2017].

PREQIN (2016): "The 2016 Preqin Global Natural Resources Report". Disponible en: https://www.preqin.com/docs/samples/2016-Preqin-Global-Natural-Resources-Report-SamplePages.pdf [consulta: 4 de marzo de 2017]. 
PREQIN (2017): “The private equity top 100". Disponible en: https://www.preqin.com/docs/reports/Preqin-Special-Report-The-Private-Equity-Top-100February-2017.pdf [consulta: 4 de marzo de 2017].

PRICEWATERHOUSECOOPERS (2016): "Mine 2016. Review of the global trends in the mining industry". Disponible en: www. pwc.com/mining [consulta: 17 de febrero 2017].

RISEBOROUGH, J. y BLAS, J. (2015): Bloomberg News. "For Glencore's next move watch agriculture rather than iron", 20 de marzo.

SALERNO, T. (2014): Third World Quarterly. "Capitalizing on the financiarization of agricultura: Cargill's land investment techniques in the Philippines", vol. 35, n ${ }^{\circ}$, p. 1709-1727.

SILVERSTEIN, KEN (2012): “A Giant Among Giants". Disponible en: http://foreignpolicy.com/2012/04/23/a-giant-among-giants/[consulta: 7 de octubre de 2016].

SIPPEL, S. R; LARDER, N. y LAWRENCE, G. (2016): Agriculture and Human Values. "Grounding the financialization of farmland: perspectives on financial actors as new land owners in rural Australia", vol. 34, n 2, p. 251-265.

SZALA, GINGER (2013): "10 top global commodity trading firms: Smart money or bad boys?". Disponible en: http://www.futuresmag.com/2013/07/25/10-top-global-commodity-trading-firmssmart-money-or-bad-boys?page $=10$ [consulta: 13 de marzo de 2017].

TIAA-CREF (2015): "Responsible Investment in Farmland". Disponible en: https://www.tiaa.org/public/pdf/C26304_2015_Farmland_Report.pdf [consulta: 10 de marzo de 2017].

TILAK, JOHN y SCUFFHAM, MATT (2017): "CPPIB plans farmland retreat: Reuters". Disponible en: https://www.pehub.com/canada/2017/4/cppib-plans-farmland-retreat-reuters/\# [consulta: 8 de mayo de 2017].

UNEPFI (UNITED NATIONS ENVIRONMENT PROGRAMME FINANCE INITIATIVE) (2012): "Responsible investment in farmland: a compendium of case studies". Disponible en: www.unpri.org/areas-of-work/implementation-support/commodities/) [consulta: 29 de abril de 2017].

USDA (DEPARTAMENTO DE AGRICULTURA DE ESTADOS UNIDOS) (2017): Foreign Agricultural Service. Disponible en: https://apps.fas.usda.gov/psdonline/app/index.html\#/app/advQuery [consulta: 22 de febrero de 2017].

VITERRA (2012): "Viterra shareholders approve Glencore acquisition". Disponible en: http://www.marketwired.com/press-release/viterra-shareholders-approve-glencore-acquisitiontsx-vt-1662666.htm [consulta: 8 de diciembre de 2016].

VITERRA (2016a): “About”. Disponible en: http://www.viterra.com.au [consulta: 7 de enero de 2016].

VITERRA (2016b): "Viterra makes long term SA rail investment". Disponible en: http://www.viterra.com.au [consulta: 7 de enero de 2016].

VITERRA (2017): “About". Disponible en: https://www.viterra.com/web/canada/home [consulta: 24 de febrero de 2017]. 